\title{
A!
}

This is an electronic reprint of the original article.

This reprint may differ from the original in pagination and typographic detail.

Vanhala, Tuomas I.; Siro, Topi; Liang, Long; Troyer, Matthias; Harju, Ari; Törmä, Päivi

\section{Topological Phase Transitions in the Repulsively Interacting Haldane-Hubbard Model}

Published in:

Physical Review Letters

DOI:

10.1103/PhysRevLett.116.225305

Published: 02/06/2016

Document Version

Publisher's PDF, also known as Version of record

Please cite the original version:

Vanhala, T. I., Siro, T., Liang, L., Troyer, M., Harju, A., \& Törmä, P. (2016). Topological Phase Transitions in the Repulsively Interacting Haldane-Hubbard Model. Physical Review Letters, 116(22), 1-6. [225305].

https://doi.org/10.1103/PhysRevLett.116.225305

This material is protected by copyright and other intellectual property rights, and duplication or sale of all or part of any of the repository collections is not permitted, except that material may be duplicated by you for your research use or educational purposes in electronic or print form. You must obtain permission for any other use. Electronic or print copies may not be offered, whether for sale or otherwise to anyone who is not an authorised user. 


\title{
Topological Phase Transitions in the Repulsively Interacting Haldane-Hubbard Model
}

\author{
Tuomas I. Vanhala, ${ }^{1,2}$ Topi Siro, ${ }^{1}$ Long Liang, ${ }^{1}$ Matthias Troyer, ${ }^{2}$ Ari Harju, ${ }^{1, *}$ and Päivi Törmä ${ }^{1,3, \dagger}$ \\ ${ }^{1}$ COMP Centre of Excellence, Department of Applied Physics, Aalto University, Helsinki, Finland \\ ${ }^{2}$ Theoretische Physik, ETH Zurich, 8093 Zurich, Switzerland \\ ${ }^{3}$ Institute for Quantum Electronics, ETH Zurich, 8093 Zurich, Switzerland
}

(Received 30 December 2015; published 2 June 2016)

\begin{abstract}
Using dynamical mean-field theory and exact diagonalization we study the phase diagram of the repulsive Haldane-Hubbard model, varying the interaction strength and the sublattice potential difference. In addition to the quantum Hall phase with Chern number $C=2$ and the band insulator with $C=0$ present already in the noninteracting model, the system also exhibits a $C=0$ Mott insulating phase, and a $C=1$ quantum Hall phase. We explain the latter phase by a spontaneous symmetry breaking where one of the spin components is in the Hall state and the other in the band insulating state.
\end{abstract}

DOI: 10.1103/PhysRevLett.116.225305

When a quantum system has two or more competing phases, exotic states can emerge in the crossover region between these. Especially interesting phenomena can be expected between topologically trivial and nontrivial phases. Three paradigm models that offer a generic platform to explore such intermediate phases are the HaldaneHubbard, the Kane-Mele-Hubbard, and the ionic Hubbard models. The existence and the nature of exotic intermediate states of matter between such phases, showing spectral features and responses of a mixed character, is a subtle and largely open question.

In the ionic Hubbard models an energy offset (staggering) $\Delta_{A B}$ between the two sites $(A$ and $B$ ) of a bipartite lattice is combined with an on-site repulsive interaction $U$. Starting from $\Delta_{A B}=U=0$, the models show a band insulator for large $\Delta_{A B}$ and a Mott insulator for strong interactions $U$. Predictions of possible intermediate states between the two insulators range from semimetals [1] and half-metals [2] to metallic [3-5] and insulating [5,6] ones, depending on the kinetic part of the Hamiltonian. Dimerized bond-ordered insulators have been shown to exist for 1D systems [7-9], but predictions for, e.g., the 2D square lattice are contradictory $[3,6]$. The band and Mott insulators in the ionic Hubbard model have been recently observed in ultracold quantum gases [10], but no information about a possible intermediate state was obtained.

In the Haldane model [11], a staggered magnetic flux threads a hexagonal lattice, endowing the noninteracting electronic bands with a finite Chern number and quantized Hall conductivity. Large staggering $\Delta_{A B}$ drives the system from this topological insulator into a trivial band insulator. The model was recently realized in ultracold gas implementations $[12,13]$. Large $U$ leads to a topologically trivial Mott insulator phase, but little is known about possible intermediate states. Mean-field studies [14-18] suggest the existence of an interesting insulator phase with $C=1$, but whether this phase survives the inclusion of correlations is so far unknown. The existence of intermediate states is an open question [19-23] also in the similar but time-reversalsymmetric Kane-Mele model [24], despite the fact that sign problem free quantum Monte Carlo (QMC) methods exist for that model.

In this Letter, we ask whether intermediate phases are possible in the Haldane-Hubbard model when the staggering $\Delta_{A B}$ and interaction $U$ are varied from zero to large values. We aim to investigate the nature of such states as well as their spectral properties, and to suggest feasible experimental realizations of the predicted phases. Importantly, mean-field theory is expected to be highly unreliable for the intermediate phases, not only due to strong interactions and low dimensionality (2D), but also because they are by definition states where orders of the surrounding phases compete. Therefore, a crucial ingredient of our study is that we apply two complementary, state-of-the-art beyond-mean-field methods. First, we perform exact diagonalization of finite-size clusters (FS ED). Exact diagonalization gives reliable information about the nature of the ground state, without any bias from an ansatz, and proves its stability against quantum fluctuations over the system size, but suffers from finite-size effects. Therefore, we also apply dynamical mean-field theory (DMFT) to prove that the predicted phase survives at the thermodynamic limit. DMFT goes beyond static mean-field (MF) theory by treating local quantum fluctuations exactly. Nonlocal quantum fluctuations are not included and the method might be biased by the choice of the order parameters. In our case, however, these weaknesses are controlled by the exact diagonalization results. For comparison, we also present $\mathrm{MF}$ results.

We write the Hamiltonian of the Haldane-Hubbard model (Fig. 1) as $H=H_{k}+H_{l}$, where $H_{l}$ is a local, on-site part and the kinetic term $H_{k}$ is given by 


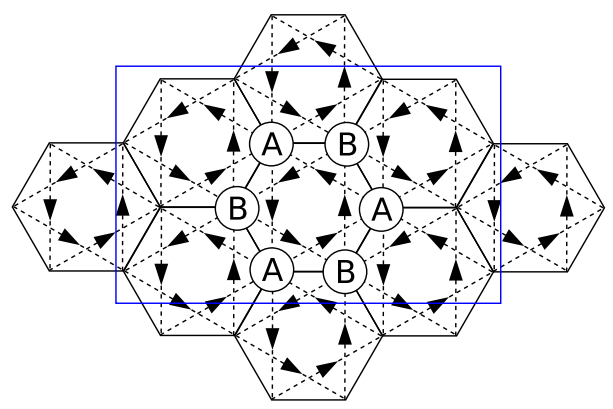

FIG. 1. A patch of the Haldane model. The model consists of a hexagonal lattice with nearest- and next-nearest-neighbor (NNN) hoppings. The arrows show the direction of positive phase winding for the complex NNN hoppings, which are responsible for the topological properties of the model. In this Letter, we study the interplay of a potential difference between sublattices $A$ and $B$ and a local Hubbard interaction. The blue rectangle shows the finite-size exact diagonalization cluster.

$$
H_{k}=t \sum_{\langle i, j\rangle, \sigma} c_{i \sigma}^{\dagger} c_{j \sigma}+t^{\prime} \sum_{\langle i, j\rangle\rangle, \sigma} \exp \left(i \phi_{i j}\right) c_{i \sigma}^{\dagger} c_{j \sigma}
$$

where $\langle i, j\rangle$ and $\langle\langle i, j\rangle\rangle$ denote summation over nearest and next-nearest neighbors on a hexagonal lattice, and $\sigma$ runs over the two spin components. The phase $\phi_{i j}$ has a constant absolute value and a sign that depends on the direction of the bond, $\phi_{i j}= \pm \phi$. The on-site part can be written as

$$
H_{l}=U \sum_{i}\left(n_{i \uparrow}-\frac{1}{2}\right)\left(n_{i \downarrow}-\frac{1}{2}\right)+\Delta_{A B} \sum_{i, \sigma} \operatorname{sgn}(i) n_{i \sigma},
$$

where $\operatorname{sgn}(i)$ is +1 for sites $i$ on sublattice $A$ and -1 for sublattice $B$. In the following, we take $t=1$ and set $t^{\prime}=0.2$. A particle-hole transformation $c_{i \sigma}^{\prime}=\operatorname{sgn}(i) c_{i \sigma}^{\dagger}$ keeps the Hamiltonian otherwise invariant, but takes $\Delta_{A B}^{\prime}=$ $-\Delta_{A B}$ and $\phi^{\prime}=\pi-\phi$. A further rotation of the lattice by $\pi$ radians around a center of a hexagon only changes the sign of $\Delta_{A B}$. We study the model at half-filling and set the phase $\phi=\pi / 2$. The above symmetries then imply that the phase diagram is symmetric under the reflection $\Delta_{A B} \rightarrow-\Delta_{A B}$ and that the chemical potential is zero. We do not consider attractive interactions which may support topological superfluids [17,25].

We study the phase diagram of this model as a function of the interaction strength $U$ and the sublattice potential difference $\Delta_{A B}$. We use an exact diagonalization impurity solver [26,27] to obtain results within single-site and twosite cellular DMFT [26,28,29], always allowing for a symmetry breaking between the $A$ and $B$ sublattices. We find that using 5 or 6 bath sites already gives a good representation of the bath Green's function. For selected parameters we have confirmed the results using the CT-INT algorithm [30,31] as the impurity solver. In the mean-field and DMFT solutions antiferromagnetism is measured by an order parameter $m$ defined as

$$
m=\frac{1}{N_{s}}\left|\sum_{i} \operatorname{sgn}(i)\left(\left\langle n_{i \uparrow}\right\rangle-\left\langle n_{i \downarrow}\right\rangle\right)\right|,
$$

where $N_{s}$ is the number of sites, and the degree to which the particles are localized to the low-energy sublattice is measured by the staggered density

$$
n_{s}=\frac{1}{N_{s}}\left|\sum_{i} \operatorname{sgn}(i)\left(\left\langle n_{i \uparrow}\right\rangle+\left\langle n_{i \downarrow}\right\rangle\right)\right| .
$$

Another important quantity is the Chern number. In [32] it was shown that knowledge of the zero-frequency Green's function is sufficient to determine topological invariants for interacting systems. The result can be formulated [33,34] by defining the so-called topological Hamiltonian as

$$
h_{t}(\vec{k}) \equiv-G(i \omega=0, \vec{k})^{-1}=h_{0}(\vec{k})+\Sigma(i \omega=0, \vec{k}),
$$

where $G$ and $\Sigma$ are the interacting single-particle Green's function and self-energy of the problem, and $h_{0}$ is the noninteracting single-particle Hamiltonian (the Bloch Hamiltonian). In our case $G, \Sigma$, and $h_{0}$ are matrices in spin and sublattice space. According to the theory, the Chern number calculated for a noninteracting problem defined by $h_{t}$ is the same as the Chern number for the original interacting problem. We obtain an approximation for the zero-frequency self-energy by a linear interpolation between the smallest-in-absolute-value Matsubara frequencies at a very low temperature, taking care that the Matsubara frequency grid is dense enough. We then calculate the topological Hamiltonian on a $k$-point grid using Eq. (5) and obtain the corresponding Bloch eigenstates. These states can be used to calculate the Chern number using the gauge invariant discretization of the Berry curvature described in [35].

The Chern number can also be calculated from FS ED using twisted boundary conditions [36],

$$
\psi\left(x_{j}+L_{j}\right)=e^{i \theta_{j}} \psi\left(x_{j}\right),
$$

where $j$ indexes the space dimensions and $L_{j}$ is the length of the system along direction $j$. The Chern number can then be calculated by dividing the $\left(\theta_{1}, \theta_{2}\right)$ plane into a discrete lattice and computing the flux of the Berry curvature from the Berry phase acquired by the state around each cell [37]. Our results have been obtained for the 16-site cluster shown in Fig. 1.

Our main result, the topological phase diagram of the model, is presented in Fig. 2. For small $U$ the main effect of the interaction is to push the transition from the quantum Hall $(\mathrm{QH})$ phase to the band insulating $(\mathrm{BI})$ phase to higher values of $\Delta_{A B}$ than in the noninteracting case. This effect can be explained in a mean-field picture: The sublattice potential difference causes a density difference between the sublattices, which in turn causes a Hartree potential that 


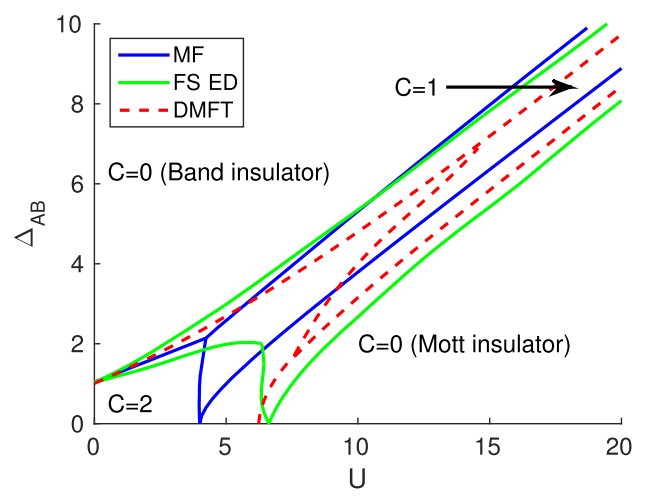

FIG. 2. The phase diagram of the model from mean-field theory (MF), finite-size exact diagonalization (FS ED) and single-site dynamical mean-field theory (DMFT). The lines indicate the topological transitions where the Chern number $C$ changes. The most interesting feature is the $C=1$ phase found by all methods between the Mott insulating and band insulating regions.

opposes this effect. The $\mathrm{QH}$ phase has Chern number $C=2$, which is the sum of the Chern numbers of the two (equivalent) spin channels.

For high interaction strengths, on the other hand, the main features of the phase diagram are the antiferromagnetic Mott insulator and the band insulator phase whose boundary roughly follows the line $\Delta_{A B}=U / 2$. Below this line $U$ dominates and the ground state of the local Hamiltonian $H_{l}$ has no doubly occupied sites, while above the line $\Delta_{A B}$ drives all of the particles to the lower energy sublattice. Nontrivial competition between the hopping, $U$ and $\Delta_{A B}$ occurs close to the line $\Delta_{A B}=U / 2$ where the large energy scales $U$ and $\Delta_{A B}$ mostly cancel each other. Indeed, we find that this boundary region between the two topologically trivial insulators exhibits a phase with Chern number $C=1$.

In the mean-field solution [14-18] for the $C=1$ phase the staggered potential drives one of the components mostly to the low-energy sublattice. Thus, this component is effectively in the topologically trivial region of the phase diagram of the Haldane model. However, the larger density of one component on the lower sublattice creates a Hartree potential that mostly cancels the sublattice potential difference $\Delta_{A B}$ for the other component, which then carries the Chern number $C=1$. Because of this symmetry breaking, the $C=1$ phase has a nonzero $m$, while the $C=2$ phase and the band insulator are paramagnetic, although the mean-field solution also has a very narrow antiferromagnetic region with $C=2$ near the $C=2$ to $C=1$ transition line.

We have confirmed this picture in the FS ED calculations by comparing the obtained ground state with an ansatz that is a symmetric linear combination $|\psi\rangle=$ $(1 / \sqrt{2})\left(|\mathrm{QH}\rangle_{\uparrow}|\mathrm{BI}\rangle_{\downarrow}+|\mathrm{BI}\rangle_{\uparrow}|\mathrm{QH}\rangle_{\downarrow}\right)$, where $|\mathrm{QH}\rangle$ and $|\mathrm{BI}\rangle$ are the single-component ground states of the noninteracting model for vanishing (quantum Hall) and large $\Delta_{A B}$ (band insulator), respectively. In Fig. 3(b) we present

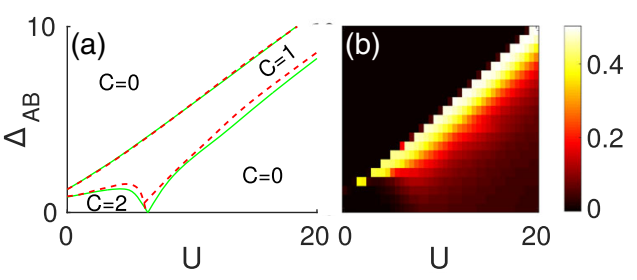

FIG. 3. (a) The single-site DMFT and FS ED phase diagrams for a finite mass imbalance. The hoppings for the up component were scaled by a factor of 0.8 , while the hoppings for the down component were scaled by 1.2 compared to the balanced situation in Fig. 2. (b) The overlap between the ground state and the trial state $|\psi\rangle$ obtained from the FS ED calculations (see text) for the same parameters as in Fig. 2.

the overlap between this state and the ground state, which reaches values as high as 0.5 in the $C=1$ region of the phase diagram. This shows that the above qualitative picture of the $C=1$ state is correct. In the FS ED results the $C=1$ phase is present already for weak interactions. However, this is a finite-size effect: A mean-field calculation for the FS ED cluster produces the same result for weak $U$, while the $C=1$ phase is absent in the infinitelattice mean field in this region. This is expected, as finitesize effects are known to be important when the band gap of the noninteracting Hamiltonian is small [38].

To further understand the nature of the $C=1$ phase we have calculated the quasiparticle gap (see Fig. 4), which in ultracold gas experiments can be studied using rf or lattice modulation spectroscopy [39]. For $U \ll 2 \Delta_{A B}$ the system is in the band insulating state and we find a gap that gets smaller as $U$ is increased. The gap has a minimum at the point where the system enters the $C=1$ state. When $U$ is increased further, the gap for the component that carries Chern number $C=1$ again reaches a minimum, and the system moves to the $C=0$ Mott insulator phase, where the gap grows as a function of $U$. In the FS ED calculation we

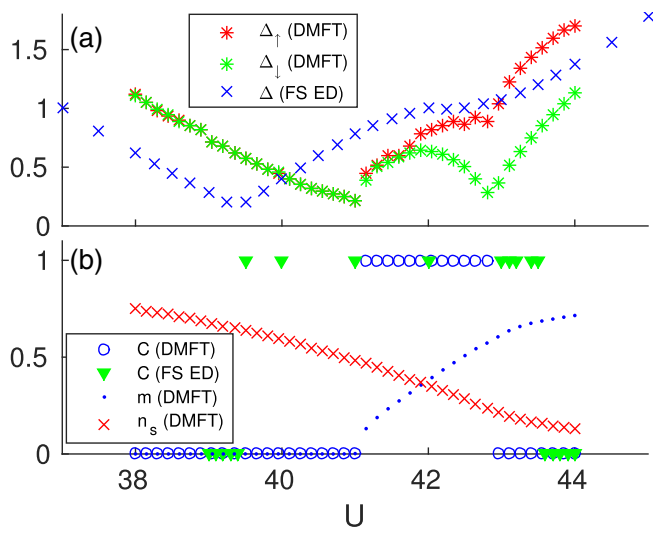

FIG. 4. (a) The quasiparticle gap $\Delta$ for the up and down components obtained from two-site DMFT and the spin-rotationinvariant result from FS ED at $\Delta_{A B}=20$. (b) The Chern number, staggered density, and the antiferromagnetic order parameter $m$ from two-site DMFT and FS ED for the same $\Delta_{A B}$. 
do not see a gap closing at the boundary of the $C=1$ phase and the Mott phase because the finite-size ground state is symmetric with respect to spin rotations.

We have also done DMFT calculations for different values of $t^{\prime}$. When $t^{\prime}$ is increased, the intermediate band between the band insulator and the Mott insulator gets wider, as the Mott insulator is pushed to larger values of $U$. At the same time the $C=2$ phase extends into higher values of $U$ within the intermediate band: for $t^{\prime}=0.3$ it is already present at values of $U>20$. However, we stress that our DMFT results for $t^{\prime} \gtrsim 0.3$ are not necessarily physical, as we have not considered, e.g., the exotic magnetic orders predicted for this parameter region [40,41].

For $\Delta_{A B}=0$ we have performed six-site cluster DMFT calculations [42] using the CT-INT impurity solver, as there is only a minor sign problem in this region. All DMFT results show a first order transition, which explains why FS ED results differ from DMFT close to $\Delta_{A B}=0$ : The finite cluster cannot exhibit a sudden development of long range order. We note that for vanishing $t^{\prime}$ the transition is indicated by large scale QMC to be of the second order [43], while cluster DMFT finds a first order transition [44]. Extrapolating to zero temperature, the existence of the antiferromagnetic solution in the dynamical cluster approximation starts at $U_{c}=6.21 \pm 0.01$, which is in good agreement with the single-site DMFT result $U_{c}=$ $6.27 \pm 0.01$, while cellular DMFT gives $U_{c}=6.6 \pm 0.1$. Wu et al. [45] find a much lower value $U_{c} \approx 3.7$ at $t^{\prime}=0.2$ in a two-site DMFT scheme. However, our results for $U_{c}$ agree well with the FS ED transition point and are consistent with the $t^{\prime}=0$ cellular DMFT result $U_{c} \approx 4.6$ [44], as $t^{\prime}>0$ is expected to increase $U_{c}$.

Finally, we note that by the Mermin-Wagner theorem the SU(2) symmetry of the model can only be broken at zero temperature. However, the critical temperatures can be made finite by adding contributions to the Hamiltonian that break the SU(2) symmetry [46,47]. A mass imbalance (i.e., different hopping strengths for the two components) would even explicitly break the whole SU(2) symmetry away from the $\Delta_{A B}=0$ line. As this enables the observation of a $C=1$ phase already for vanishing interactions, it would provide a way to test detection techniques in the noninteracting limit. Figure 3(a) presents the DMFT phase diagram of the model for a finite mass imbalance.

In summary, we found firm evidence for a $C=1$ insulator in the Haldane-Hubbard model which spontaneously breaks the $\mathrm{SU}(2)$ spin-rotation symmetry of the model. Our results differ from the mean-field result [14-18] which sets the $\Delta_{A B}=0$ boundary between the $C=2$ and Mott insulators at $U=4 t$, and predicts the $C=1$ phase to be present for small $\Delta_{A B}$. In contrast, we find the boundary at $U=6 t$ and that the $C=1$ phase is more likely to occur only for $\Delta_{A B} \gtrsim 2$ as predicted by DMFT (the prediction of this phase by FS ED for small $\Delta_{A B}$ is likely a finite-size effect). It is also not clear if the slave-spin theory of [18] is an improvement over the mean-field treatment as it, e.g., finds that the critical $U$ for antiferromagnetism at the $\Delta_{A B}=0$ line is decreased compared to the mean-field result, and there is an unphysical first order transition to the Mott phase with $n_{s}$ as the order parameter. The variational cluster ansatz results $[45,48]$ differ from ours in that they exhibit a topological Mott insulator phase and a narrow topological antiferromagnetic region for $\Delta_{A B}=0$, which are not found in our FS ED or DMFT calculations. We also do not see signs of spin liquid phases predicted to occur $[15,49]$ already for small $t^{\prime}$. However, our study does not exclude the possibility of spin liquids or exotic magnetic order predicted $[14,40,41,50,51]$ for $t^{\prime} \gtrsim 0.3$, which strongly frustrates the simple antiferromagnetic order and thus enables other phases to be present.

In comparison to the ionic Hubbard models, the intermediate state we found is more robust and occupies a larger part of the phase diagram. For instance, the intermediate insulator state in [6] vanishes around $U=11$ while ours continues. In [1,4], large $\Delta_{A B}$ suppresses the intermediate phase while in our case it helps to stabilize it. The halfmetal found in [2], which resembles our $C=1$ state since only one spin component is gapped, exists in a tiny parameter regime compared to the large stability area we find. Thus, it seems evident that the intermediate phase in the Haldane-Hubbard model, compared to the ionic Hubbard one, is stabilized by topological effects. In contrast to the semimetal of the ionic Hubbard model, the phase diagram starts from $\Delta_{A B}=U=0$ as a topological insulator with $C=2$. Characteristics of such an insulator, for one component, are inherited in the $C=1$ phase. From the comparison to the mean-field studies of the Haldane-Hubbard model and to the ionic Hubbard model results we conclude that while correlation effects tend to destroy the $C=1$ phase for small $\Delta_{A B}$, it survives as an exceptionally stable intermediate state close to the $\Delta_{A B}=U / 2$ line in the very strongly interacting region.

Experimental observation of the predicted phases would be of fundamental importance for understanding not only the Haldane-Hubbard model but also the intermediate states in its cousin models. We calculate the quasiparticle gap, and suggest that it could be used for probing the phase diagram experimentally. Finally, we propose that a finite mass imbalance would make it easier to experimentally access the interesting features of the phase diagram.

We thank T. Esslinger, G. Jotzu, R. Desbuquois, M. Messer, and F. Görg for useful discussions. This work was supported by the Academy of Finland through its Centers of Excellence Programme (2012-2017) and under Projects No. 263347, No. 251748, No. 284621, and No. 272490, by the European Research Council (ERC-2013-AdG-340748CODE and ERC-2011-AdG-290464-SIMCOFE), by the Swiss National Science Foundation through the National Competence Center in Research QSIT, and by the Pauli Center for Theoretical Studies at ETH Zurich. T. I. V. is 
grateful for the support from the Vilho, Yrjö, and Kalle Väisälä Foundation. T. S. acknowledges financial support from the Finnish Doctoral Programme in Computational Sciences FICS. Computing resources were provided by CSC - the Finnish IT Centre for Science and the Triton cluster at Aalto University.

*ari.harju@aalto.fi paivi.torma@aalto.fi

[1] M. Ebrahimkhas, Z. Drezhegrighash, and E. Soltani, Effects of correlations on honeycomb lattice in ionic-Hubbard model, Phys. Lett. A 379, 1053 (2015).

[2] S. Bag, A. Garg, and H. R. Krishnamurthy, Phase diagram of the half-filled ionic Hubbard model, Phys. Rev. B 91, 235108 (2015).

[3] N. Paris, K. Bouadim, F. Hebert, G. G. Batrouni, and R. T. Scalettar, Quantum Monte Carlo Study of an InteractionDriven Band-Insulator-to-Metal Transition, Phys. Rev. Lett. 98, 046403 (2007).

[4] A. Garg, H. R. Krishnamurthy, and M. Randeria, Can Correlations Drive a Band Insulator Metallic? Phys. Rev. Lett. 97, 046403 (2006).

[5] K. Byczuk, M. Sekania, W. Hofstetter, and A. P. Kampf, Insulating behavior with spin and charge order in the ionic Hubbard model, Phys. Rev. B 79, 121103 (2009).

[6] S. S. Kancharla and E. Dagotto, Correlated Insulated Phase Suggests Bond Order between Band and Mott Insulators in Two Dimensions, Phys. Rev. Lett. 98, 016402 (2007).

[7] M. Fabrizio, A. O. Gogolin, and A. A. Nersesyan, From Band Insulator to Mott Insulator in One Dimension, Phys. Rev. Lett. 83, 2014 (1999).

[8] T. Wilkens and R. M. Martin, Quantum Monte Carlo study of the one-dimensional ionic Hubbard model, Phys. Rev. B 63, 235108 (2001).

[9] C. D. Batista and A. A. Aligia, Exact Bond Ordered Ground State for the Transition between the Band and the Mott Insulator, Phys. Rev. Lett. 92, 246405 (2004).

[10] M. Messer, R. Desbuquois, T. Uehlinger, G. Jotzu, S. Huber, D. Greif, and T. Esslinger, Exploring Competing Density Order in the Ionic Hubbard Model with Ultracold Fermions, Phys. Rev. Lett. 115, 115303 (2015).

[11] F. D. M. Haldane, Model for a Quantum Hall Effect without Landau Levels: Condensed-Matter Realization of the "Parity Anomaly," Phys. Rev. Lett. 61, 2015 (1988).

[12] G. Jotzu, M. Messer, R. Desbuquois, M. Lebrat, T. Uehlinger, D. Greif, and T. Esslinger, Experimental realization of the topological Haldane model with ultracold fermions, Nature (London) 515, 237 (2014).

[13] N. Fläschner, B. S. Rem, M. Tarnowski, D. Vogel, D. S. Lühmann, K. Sengstock, and C. Weitenberg, Experimental reconstruction of the Berry curvature in a topological Bloch band, arXiv:1509.05763.

[14] J. He, Y.-H. Zong, S.-P. Kou, Y. Liang, and S. Feng, Topological spin density waves in the Hubbard model on a honeycomb lattice, Phys. Rev. B 84, 035127 (2011).

[15] J. He, Y. Liang, and S.-P. Kou, Composite spin liquid in a correlated topological insulator: Spin liquid without spincharge separation, Phys. Rev. B 85, 205107 (2012).
[16] Y.-X. Zhu, J. He, C.-L. Zang, Y. Liang, and S.-P. Kou, Magnetic topological insulators at finite temperature, J. Phys. Condens. Matter 26, 175601 (2014).

[17] Y.-J. Wu, N. Li, and S.-P. Kou, Chiral topological superfluids in the attractive Haldane-Hubbard model with opposite Zeeman energy at two sublattice sites, Eur. Phys. J. B 88, 255 (2015).

[18] D. Prychynenko and S. Huber, $Z_{2}$ slave-spin theory of a strongly correlated Chern insulator, Physica B (Amsterdam) 481, 53 (2016).

[19] H.-H. Hung, V. Chua, L. Wang, and G. A. Fiete, Interaction effects on topological phase transitions via numerically exact quantum Monte Carlo calculations, Phys. Rev. B 89, 235104 (2014).

[20] Y.-H. Zong, J. He, and S.-P. Kou, Quantum spin liquid in interacting Kane-Mele model with staggered on-site potential, Eur. Phys. J. B 86, 28 (2013).

[21] D. Pesin and L. Balents, Mott physics and band topology in materials with strong spin-orbit interaction, Nat. Phys. 6, 376 (2010).

[22] M. Hohenadler, Z. Y. Meng, T. C. Lang, S. Wessel, A. Muramatsu, and F. F. Assaad, Quantum phase transitions in the Kane-Mele-Hubbard model, Phys. Rev. B 85, 115132 (2012).

[23] Y.-H. Chen, H.-H. Hung, G. Su, G. A. Fiete, and C. S. Ting, Cellular dynamical mean-field theory study of an interacting topological honeycomb lattice model at finite temperature, Phys. Rev. B 91, 045122 (2015).

[24] C. L. Kane and E. J. Mele, Quantum Spin Hall Effect in Graphene, Phys. Rev. Lett. 95, 226801 (2005).

[25] Y. C. Zhang, Z. Xu, and S. Zhang, Topological superfluids and BEC-BCS crossover in attractive Haldane-Hubbard model, arXiv:1511.03833.

[26] A. Georges, G. Kotliar, W. Krauth, and M. J. Rozenberg, Dynamical mean-field theory of strongly correlated fermion systems and the limit of infinite dimensions, Rev. Mod. Phys. 68, 13 (1996).

[27] M. Caffarel and W. Krauth, Exact Diagonalization Approach to Correlated Fermions in Infinite Dimensions: Mott Transition and Superconductivity, Phys. Rev. Lett. 72, 1545 (1994).

[28] T. Maier, M. Jarrell, T. Pruschke, and M. H. Hettler, Quantum cluster theories, Rev. Mod. Phys. 77, 1027 (2005).

[29] G. Kotliar, S. Y. Savrasov, G. Pálsson, and G. Biroli, Cellular Dynamical Mean Field Approach to Strongly Correlated Systems, Phys. Rev. Lett. 87, 186401 (2001).

[30] A. N. Rubtsov, V. V. Savkin, and A. I. Lichtenstein, Continuous-time quantum Monte Carlo method for fermions, Phys. Rev. B 72, 035122 (2005).

[31] E. Gull, A. J. Millis, A. I. Lichtenstein, A. N. Rubtsov, M. Troyer, and P. Werner, Continuous-time Monte Carlo methods for quantum impurity models, Rev. Mod. Phys. 83, 349 (2011).

[32] Z. Wang and S.-C. Zhang, Simplified Topological Invariants for Interacting Insulators, Phys. Rev. X 2, 031008 (2012).

[33] Z. Wang and B. Yan, Topological Hamiltonian as an exact tool for topological invariants, J. Phys. Condens. Matter 25, 155601 (2013).

[34] W. Witczak-Krempa, M. Knap, and D. Abanin, Interacting Weyl Semimetals: Characterization via the Topological 
Hamiltonian and Its Breakdown, Phys. Rev. Lett. 113, 136402 (2014).

[35] T. Fukui, Y. Hatsugai, and H. Suzuki, Chern numbers in discretized Brillouin zone: Efficient method of computing (spin) Hall conductances, J. Phys. Soc. Jpn. 74, 1674 (2005).

[36] Q. Niu, D. J. Thouless, and Y.-S. Wu, Quantized Hall conductance as a topological invariant, Phys. Rev. B 31, 3372 (1985).

[37] R. Resta, The insulating state of matter: A geometrical theory, Eur. Phys. J. B 79, 121 (2011).

[38] C. N. Varney, K. Sun, M. Rigol, and V. Galitski, Topological phase transitions for interacting finite systems, Phys. Rev. B 84, 241105 (2011).

[39] P. Törmä, Spectroscopies-theory, in Quantum Gas Experiments-Exploring Many-Body States, edited by P. Törmä and K. Sengstock (Imperial College Press, London, 2015).

[40] C. Hickey, L. Cincio, Z. Papić, and A. Paramekanti, Haldane-Hubbard Mott Insulator: From Tetrahedral Spin Crystal to Chiral Spin Liquid, Phys. Rev. Lett. 116, 137202 (2016).

[41] C. Hickey, P. Rath, and A. Paramekanti, Competing chiral orders in the topological Haldane-Hubbard model of spin1/2 fermions and bosons, Phys. Rev. B 91, 134414 (2015).

[42] A. Liebsch and W. Wu, Coulomb correlations in the honeycomb lattice: Role of translation symmetry, Phys. Rev. B 87, 205127 (2013).
[43] S. Sorella, Y. Otsuka, and S. Yunoki, Absence of a spin liquid phase in the Hubbard model on the honeycomb lattice, Sci. Rep. 2, 992 (2012).

[44] R.-Q. He and Z.-Y. Lu, Cluster dynamical mean field theory of quantum phases on a honeycomb lattice, Phys. Rev. B 86, 045105 (2012).

[45] J. Wu, J. P. L. Faye, D. Sénéchal, and J. Maciejko, Quantum cluster approach to the spinful Haldane-Hubbard model, Phys. Rev. B 93, 075131 (2016).

[46] A. Cuccoli, T. Roscilde, V. Tognetti, R. Vaia, and P. Verrucchi, Quantum Monte Carlo study of $s=\frac{1}{2}$ weakly anisotropic antiferromagnets on the square lattice, Phys. Rev. B 67, 104414 (2003).

[47] A. Cuccoli, T. Roscilde, R. Vaia, and P. Verrucchi, Fieldinduced $X Y$ behavior in the $S=\frac{1}{2}$ antiferromagnet on the square lattice, Phys. Rev. B 68, 060402 (2003).

[48] Z. L. Gu, K. Li, and J. X. Li, Topological phase transitions and topological Mott insulator in Haldane-Hubbard model, arXiv:1512.05118.

[49] J. He, S.-P. Kou, Y. Liang, and S. Feng, Chiral spin liquid in a correlated topological insulator, Phys. Rev. B 83, 205116 (2011).

[50] X.-J. Liu, Z.-X. Liu, K. T. Law, W. V. Liu, and T. K. Ng, Chiral topological orders in an optical Raman lattice, New J. Phys. 18, 035004 (2016).

[51] W. Zheng, H. Shen, Z. Wang, and H. Zhai, Magnetic-orderdriven topological transition in the Haldane-Hubbard model, Phys. Rev. B 91, 161107 (2015). 